\title{
Author Index Volume 5 (1997)
}

The issue number is given in front of the page numbers.

Amouroux, R., see Bryce, C.

Asokan, N., G. Tsudik and M. Waidner, Server-supported signatures

Atluri, V. and W.-K. Huang, Enforcing mandatory and discretionary

(3) $225-235$

security in workflow management systems

(1) $91-108$

(4) $303-339$

Bergadano, F., B. Crispo and M. Lomas, Strong authentication and privacy with standard browsers

Bonatti, P.A., M.L. Sapino and V.S. Subrahmanian, Merging heterogeneous security orderings

Bryce, C., W. Kühnhauser, R. Amouroux, M. Lopez and H. Rudnik, CWASAR: a European infrastructure for secure electronic commerce

Camenisch, J., U. Maurer and M. Stadler, Digital payment systems with passive anonymity-revoking trustees

Castano, S., S. De Capitani di Vimercati and M.G. Fugini, Automated derivation of global authorizations for database federations

Ching, N., see Winslett, M.

Crispo, B., see Bergadano, F.

(3) $191-212$

(1) $3-29$

(3) $225-235$

De Capitani di Vimercati, S. and P. Samarati, Authorization specification and enforcement in federated database systems

De Capitani di Vimercati, S., see Castano, S.

Demurjian, Sr., S.A. and T.C. Ting, Towards a definitive paradigm for security in object-oriented systems and applications

(1) $69-89$

(4) $271-301$

(3) $255-267$

(3) $191-212$

Fugini, M.G., see Castano, S.

(2) $155-188$

(4) $271-301$

(4) $341-382$

(4) $271-301$

Goldschlag, D.M., see Syverson, P.F.

(3) $237-248$

Huang, W.-K., see Atluri, V.

(4) $303-339$

Jones, V., see Winslett, M.

(3) $255-267$

Joye, M., see Quisquater, J.-J.

(3) $213-223$

Kühnhauser, W., see Bryce, C.

(3) $225-235$

Lomas, M., see Bergadano, F.

(3) $191-212$

López, M., see Bryce, C.

(3) $225-235$

Lotz, V., Threat scenarios as a means to formally develop secure systems

(1) $31-67$

0926-227X/97/\$8.00 @ 1997 - IOS Press. All rights reserved 
Malkhi, D. and M. Reiter, A high-throughput secure reliable multicast protocol

(2) $113-127$

Maurer, U., see Camenisch, J.

(1) $69-89$

Quisquater, J.-J. and M. Joye, Authentication of sequences with the $\mathrm{SL}_{2}$ hash function: application to video sequences

(3) $213-223$

Reed, M.G., see Syverson, P.F.

(3) $237-248$

Reiter, M., see Malkhi, D.

Röscheisen, M. and T. Winograd, A network-centric design for relationship-based security and access control

(2) $113-127$

Rudnik, H., see Bryce, C.

(3) $249-254$

(3) $225-235$

Samarati, P., see De Capitani di Vimercati, S.

(2) $155-188$

Sapino, M.L., see Bonatti, P.A.

Sinclair, J., Action systems for security specification

(1) $3-29$

(2) $129-154$

(3) $255-267$

(1) $69-89$

Stadler, M., see Camenisch, J.

Subrahmanian, V.S., see Bonatti, P.A.

(1) $3-29$

Syverson, P.F., M.G. Reed and D.M. Goldschlag, Private Web browsing

(3) $237-248$

Ting, T.C., see Demurjian, Sr., S.A.

(4) $341-382$

Tsudik, G., see Asokan, N.

(1) $91-108$

Waidner, M., see Asokan, N.

(1) $91-108$

Winograd, T., see Röscheisen, M.

Winslett, M., N. Ching, V. Jones and I. Slepchin, Using digital creden-

(3) $249-254$ tials on the World Wide Web

(3) $255-267$ 